Medienaktivitäten oder die Mini-Legislaturen der Rundfunkräte. Sie sind aber unzweifelhaft entscheidende Impulsgeber für Medienpolitik, so wie sie sich in aller Widersprüchlichkeit später darstellt. Akteure in der Medienpolitik sind eben nicht nur (und nicht einmal primär) die Aufsichtsbehörden im Rundfunk, die in diesem Buch einen eigenen Abschnitt erhalten.

Ebenso fällt die Zivilgesellschaft mit ihren Non-Government-Organisationen und dem Anspruch, selbst Medien betreiben zu wollen, trotz allen Governance-Bezugs weitgehend heraus. Sie werden vor allem als Nutzer perzipiert (im Beitrag von Hasebrink), aber im Kontext einer aktiven Demokratie müssen sie auch als handelnde Bürger ihren Ort finden. Dies wäre unter Einbezug von Varianten der Governance-Theorie bestens zu bewerkstelligen. So etablierte Ex-UN-Generalsekretär Kofi Annan eine „Working Group on Internet Governance" (2003-2005), in der die drei Bänke Staat, Wirtschaft und Zivilgesellschaft gleichgewichtig besetzt wurden, die sich über die Zukunft der vernetzten Kommunikation Gedanken machen sollten. Nach entsprechenden Modellen ließe sich auch die Verbindung von Theorie und Praxis im politischen InputBereich organisieren.

Dieser Sammelband vereint viele Beiträge, die sich bewusst mit ordnungspolitischen Positionen innerhalb der Kommunikationswissenschaft identifizieren, andere widersprechen dieser Sichtweise zumindest nicht. Juristen und Ökonomen sind an der Detailarbeit beteiligt, aktuelle politikwissenschaftliche Sichtweisen - auch wenn Jarren und Donges aus dieser Disziplin kommen - werden weniger beachtet. Ebenso ist die komparative Perspektive im Ansatz stecken geblieben, was z. B. erst die behauptete Nähe von Regulierungstheorie zu Medienpolitik ermöglicht, denn das gäbe so im angloamerikanischen Herkunftsgebiet des regulation-Begriffs wenig Sinn (Saxer 22ff.).

Die hier präsentierte ordnungspolitische Herangehensweise - man kann fast von einer Züricher Schule sprechen - wird in bisher nicht erreichter Klarheit vorgestellt, insofern eignet sich der Titel auch für den Einsatz in der Lehre. Die kritische Dimensionierung müssen dann wohl andere Autoren übernehmen.

Hans J. Kleinsteuber

\section{Klaus Peter Treumann et al.}

\section{Medienhandeln Jugendlicher}

Mediennutzung und Medienkompetenz

Wiesbaden: VS Verlag für Sozialwissenschaften, 2007. $-808 \mathrm{~S}$.

ISBN 978-3-531-15293-6

Wie kaum ein anderes Thema ist „Jugend und Medien" wissenschaftlich bearbeitet worden und wird es auch derzeit - wie es zuerst die kontinuierlichen Shell-Jugendstudien und die Jugendmedienstudien (JIM) des Medienpädagogischen Forschungsverbunds Südwest, aber auch viele Einzelstudien des DJF, JFF und des Hans-Bredow-Instituts belegen. Dabei ist sicherlich zu berücksichtigen, dass sowohl der Begriff der Jugend seit seiner Entstehung Anfang des 20. Jahrhunderts als auch und erst recht der der Medien infolge der technologischen Entwicklungen enorme Veränderungen erfahren haben. Aber spätestens seit den Zürcher Jugendstudien Anfang der 80er Jahre sind die sachlich-theoretischen Prämissen einigermaßen geklärt, und die erste umfassende Studie $\mathrm{zu}$ "Jugend und Medien“ der ARD/ZDF-Medienkommission und der Bertelsmann-Stiftung von 1986, die sich erstmals erklärtermaßen einem funktionalistischen Forschungsverständnis verschrieb, verdichtete Werthaltungen von Jugendlichen zu typischen Clustern und ordnete ihnen Mediennutzungs- und Medienimages zu.

Wenn jetzt vornehmlich aus Bielefeld eine neuerliche, über 800 klein gedruckte Seiten starke Studie zum „Medienhandeln Jugendlicher" (12 bis 20 Jahre) vorgelegt wird, die das Ergebnis eines gut ausgestatteten DFG-Forschungsprojektes an den Universitäten Bielefeld, Rostock und Halle-Wittenberg zwischen 2001 und 2004 ist, dann hätte man wohl angesichts der Forschungslage mindestens zweierlei erwarten dürfen: zunächst eine gründliche und umfassende Aufarbeitung dieses Forschungsstandes, um wichtige sachliche wie theoretische Desiderate zu identifizieren und die Forschung tatsächlich voranzubringen. In diesem Kontext könnten dazu etwa zählen: zum einen die Erfassung der speziellen, Peergroup bezogenen, kontextuellen Mediennutzungsformen von Jugendlichen wie Disco, Kino, LAN-Partys etc., die sich besonders mit den digitalen, interaktiven Medien als Plattformen, OnlineNetzwerken und social communities rasant 
und erheblich weiterentwickeln, verstärken und diversifizieren; zum andern die entwicklungs- und sozialisationsbezogene Dynamisierung und soziokulturelle Konkretisierung des Allerweltsbegriff der Medienkompetenz, wie sie Kinder und Jugendliche im Laufe ihres Aufwachsens sicherlich ganz unterschiedlich ausbilden. Dieser Begriff wurde Anfang der 80er Jahre eher zufällig vom verstorbenen Mentor der Bielefelder Medienpädagogik, Dieter Baacke, konzipiert und erst allmählich ausformuliert, inzwischen ist er vielfach kritisiert, modifiziert, erweitert und just auf das Fehlen der genannten Aspekte hin gerügt worden, obwohl ihn nun Baackes Kollegen und Schüler in diesem Band als ein „Modell“ kanonisieren und die seither geführten Diskussionen darüber gänzlich ignorieren. Auch generell ersparen sie sich die erforderliche und weiterführende Aufarbeitung der Jugendmedienforschung nicht, wie sie auch Ansätze der Medienforschung, wie der Uses and Gratifications-Approach, die Medienökologie, die Wissenskluft-Hypothese und endlich Bourdieus Konzept des kulturellen Kapitals, kursorisch und relativ beliebig nebeneinander stellen; selbst auf die frühere, von einigen von ihnen erarbeitete Bielefelder Studie "Lebenswelten Jugendlicher" von 1990 nehmen sie nur gelegentlich Bezug, so dass sie keine Entwicklungs- und Veränderungslinien skizzieren können.

Sodann hätte man sich methodische Innovationen versprechen können, um die enorme Komplexität, aber auch Kontingenz des kontextuellen, sozial eingebundenen und medial konvergenten Medienhandelns Jugendlicher analytisch orten und empirisch erfassen zu können. Dafür werden in dieser Studie eine breite quantitative Befragung von $3.200 \mathrm{Ju}-$ gendlichen, 40 leitfadengestützte Interviews und zehn Gruppendiskussionen zu einem speziellen Medienereignis, nämlich dem Anschlag auf das New Yorker World Trade Center und seine mediale Präsentation im September 2001, kombiniert: „Triangulation“ heißt das „methodologische Leitbild“, und insbesondere die quantitativen Daten werden ausführlichen methodologischen und stochastischen Erörterungen unterzogen, so dass sich auf vielen Seiten gewissermaßen eine ausgefeilte Lehrlektion findet. Weniger dagegen werden Fragen der sachlichen Validität erörtert: nämlich etwa die, ob die vorgelegten Items zur Erfassung von Medienkompetenz geeignet und aus- reichend sind, oder auch die, ob es nicht zu zirkulären, mindestens konformen Ergebnissen führt, wenn die Jugendlichen, die bei der Clusteridentifikation als besonders typisch ausfallen, zum qualitativen Interview herangezogen werden und dadurch möglicherweise untypische oder gar abweichende Mediennutzungsweisen ausgesondert werden. Auch bleiben die Gruppendiskussionen zu 9/11 recht marginal und lassen die methodologisch geforderte Einbindung vermissen. Und neuere Methoden wie Netzwerkanalysen werden nicht in Betracht gezogen.

Medienhandeln Jugendlicher wird ohne eine handlungstheoretische Begründung eigentlich recht lapidar als Kombination von Mediennutzung und Medienkompetenz (wie in Bielefeld verstanden) definiert, und dadurch bleiben jene typischen jugendlichen Mediennutzungsformen a priori aus dem systematischen Blickfeld und werden auch in der quantitativen Erhebung nicht erschöpfend erfasst. Hingegen tauchen sie dann plötzlich in den qualitativen Interviews auf und beschreiben dort schon maßgeblich jugendliches Medienhandeln, so dass sie auch in die Typisierungen einfließen. Ende 2001 wurde die angeblich für die Bundesländer Nordrhein-Westfalen, Mecklenburg-Vorpommern und Sachsen-Anhalt repräsentative Befragung der 12- bis 20-Jährigen durchgeführt. Von Seiten des Fragebogens fällt sie sehr ausführlich aus, und von der Zahl der befragten Jugendlichen ist sie beeindruckend. Indes erscheinen die nun dargestellten Befunde im Jahr 2007 angesichts des rasanten Wandelns der Medientechnologie und -nutzung schon ein wenig historisch und führen trotz ihrer Fülle und Differenziertheit nicht über die Daten der JIM-Studien hinaus. Für die nun erst vorgenommene Publikation wäre es sicherlich aufschlussreich gewesen, die Daten mit denen der JIM-Studien zu vergleichen und Übereinstimmungen und Abweichungen zu markieren, zumal die Autoren die JIM-Studien von 2001 bis 2004 in ihrer Literaturliste aufführen.

Aus diesen Nutzungs- und Kompetenzdaten generieren die Autoren sieben Cluster: die „Bildungsorientierten“ (in der abschließenden Zusammenfassung auch ohne Erklärung als die „schlauen Engagierten“ bezeichnet) mit 20,4\%, die „Positionslosen (oder „unkritischen Naiven“) mit 20,3\%, die „Kommunikationsorientierten“ (oder „unbedarften Integrierten“) mit 19,1\%, die „Konsumorien- 
tierten“ (oder "pragmatischen Hedonisten“) mit 17,4 \%, die „Allrounder“ (oder „versierten Avantgardisten“) mit $12 \%$, die „Deprivierten“ (oder „unauffälligen Einzelgänger“) mit $7,8 \%$ und die "Gestalter“ (oder „kritischen Macher") mit 3,1 \% - über die gewählten und am Ende ergänzten Bezeichnungen lässt sich sicherlich streiten. Aber da inzwischen fast alle Mediennutzungsstudien, nicht nur im Jugendbereich, mit solchen Typisierungen aufwarten, wäre es im Sinne der Vergleichbarkeit hilfreich gewesen, ihren Aussagegehalt kritisch abzuwägen. Salopp formuliert: So viele Typen jugendlichen Verhaltens kann es eigentlich gar nicht geben, wie die Forschungen mittlerweile kreiert haben.

Als Schwerpunkte arbeitet die Studie die ausführlichen 40 Porträts von Jugendlichen und ihrem Umgang mit Medien im Rahmen der vorgenommenen Clusterung heraus, und zwar sowohl als einzelne Fallstudien wie auch als Clusterprofile, was oft zu Redundanzen führt. Erneut illustrieren diese Profile, wie unterschiedlich das Medienhandeln ausfällt, wie es aber auch in die jeweiligen Alltage und Lebenswelten funktional und selbstständig eingebunden und umgekehrt von den soziokulturellen Kontexten wie Fähigkeiten, dem „kulturellen Kapital“, konstituiert wird. Da kann diese Bielefelder Studie gewiss noch einige differenzierende und konkretisierende Facetten zu dem nun schon erheblichen Fundus an Einsichten und Materialien hinzufügen. Die nach wie vor offene Frage, wie sich Medienhandeln und -kompetenz im Laufe der jugendlichen Entwicklung und Sozialisation gerade bei unterschiedlichen soziokulturellen Bedingungen herausbildet, wo also welche Optionen und wo welche Defizite entstehen, kann die Bielefelder Studie als einmalige Querschnittserhebung leider auch nicht beantworten, obwohl ihre altersmäßig weit spannende Population von 12 bis 20 Jahren, in denen in der jugendlichen Entwicklung bekanntlich viel passiert, dazu nachgerade eine fundierte Antwort verlangt.

Hans-Dieter Kübler

\section{Liesbet van Zoonen}

\section{Entertaining the citizen}

When politics and popular culture converge Lanham: Rowman \& Littlefield Publishers, 2005. - $192 \mathrm{~S}$.

\section{ISBN 978-0742529076}

Fingerschnippend wippt der deutsche Außenminister Frank-Walter Steinmeier gemeinsam mit seinem französischen Kollegen im Studio des deutsch-türkischen Sängers Muhabbet. Erfolgreich nutzt er das Mikrophon dieses Mal ein wenig anders als sonst, um erfolgreiche Integrationspolitik sichtbar zu machen. „Deutschland - warum verschließt Du Dich?" rappen die beiden älteren Herren. Ein erfolgreiches Polit-Event, bei dem sie der Aufmerksamkeit der versammelten Medienvertreter sicher sind. Und ihrer spöttischen Ironie zugleich. „Ode an die Freunde“, titelt die Süddeutsche Zeitung am nächsten Tag.

Für Liesbet van Zoonen dürfte Steinmeiers Auftritt ein exemplarisches Ereignis darstellen für eine Öffentlichkeit, in der Politik und Populärkultur konvergiert. (Auch wenn er sich dabei ein wenig unbeholfener anstellt als Bill Clinton mehr als 15 Jahre zuvor.) Aber im Unterschied zu den Politikredakteuren der SZ sieht sie darin nicht allein eine Bedrohung und Beeinträchtigung demokratischer Öffentlichkeit. Sie interessiert sich für die Potenziale und Optionen für die Bürgerinnen und Bürger, die in der wechselseitigen Beeinflussung von Pop-Musik, Soap-Opera und dem Handeln von Politikern liegt. Weil diese Sichtweise in der deutschsprachigen Forschung äußerst rar ist, ist ihr Buch lesens- und diskutierenswert, auch für diejenigen, die nicht allen Annahmen zustimmen.

Van Zoonen analysiert die Konstituierung von citizenship durch Populärkultur. In Anlehnung an Thomas Marshall verweist sie dabei auf die Drei- (aktuell Vier-)Teilung von political, social, civic und aktuell ergänzend cultural citizenship. Sie beschränkt sich bei ihren Analysen jedoch auf die enge, originär politische Dimension von citizenship. Und ihre zentrale Ausgangsthese lautet, dass eben diese politische Staatsbürgerschaft maßgeblich durch populärkulturelle Ausdrucksformen geprägt und gestaltet wird.

Damit reichen ihre Überlegungen über die 